East African Medical Journal Vol. 83 No. 1 January 2006

INFERTILITY IN A COMMUNITY AND CLINIC-BASED SAMPLE OF COUPLES IN MOSHI, NORTHERN TANZANIA

U. Larsen, PhD, Department of Population and International Health, Harvard School of Public Health, 665 Huntington Avenue, Boston, MA 02115, USA, G. Masenga MD, Department of Obstetrics and Gynaecology, Kilimanjaro Christian Medical Centre, P.O. Box 3010, Moshi, Tanzania and J. Mlay, MD, Department of Obstetrics and Gynaecology, Mbeya Referral Hospital, Mbeya, Tanzania

Request for reprints to: Dr. U. Larsen, Department of Population and International Health, Harvard School of Public Health, 665 Huntington Avenue, Boston, MA 02115, USA

\title{
INFERTILITY IN A COMMUNITY AND CLINIC-BASED SAMPLE OF COUPLES IN MOSHI, NORTHERN TANZANIA
}

\author{
U. LARSEN, G. MASENGA and J. MLAY
}

\begin{abstract}
Background: Previous research on the aetiology of infertility in sub-Saharan Africa was generally clinic based and it is not known whether findings from this work are representative of the general population. A better understanding of the medical causes of infertility is crucial for reducing the incidence of infertility and for improving the clinical management.

Objective: To determine the type and aetiology of infertility in a community and clinicbased sample.

Design: Couples identified as infertile in a representative cross-sectional survey from a community-based sample of 2019 women aged 20-44 years and couples seeking care for infertility at a tertiary health facility.

Setting: The community-based sample was drawn from Moshi Urban District and the clinic-based sample from patients seeking care at Kilimanjaro Christian Medical Centre (KCMC) in 2002 and 2003.

Participants: Sixty six couples identified as infertile in the community-based sample and 112 couples seeking care for infertility.

Results: The percentage of primary infertility was $37.1 \%$ and secondary infertility was $62.9 \%$. Female only factor infertility was identified in $65.9 \%$ of the couples, male only factor in $\mathbf{6 . 8 \%}$, male and female factors in $15.2 \%$ and unexplained infertility in $12.1 \%$. Conclusion: The type and aetiology of infertility were the same in the community and clinic-based sample suggesting that the couples seeking infertility health care were representative of the general infertile population. Tubal factor infertility was the commonest cause.
\end{abstract}

\section{INTRODUCTION}

At the brink of the 21 st century there was a surge in research on the social consequences and psychological disorders of infertility in non-western societies (1-3). This heightened awareness and research interest in infertility of pathological origin was rooted in the emergence of new reproductive technologies in nonwestern societies, e.g. Egypt (4). Sub-Saharan Africa carries the biggest burden of infertility worldwide (5). Even so, this region has the least developed capacity for infertility treatment, and new reproductive technologies, such as in vitro fertilization (IVF) and intra-cytoplasmic sperm injection (ISCI) have yet only been applied successfully in a few countries, such as Nigeria and South Africa $(6,7)$. To date, there is very limited knowledge about the medical causes of infertility in sub-Saharan Africa, and such data are urgently needed to improve the clinical management of infertile couples, as well as to prevent onset of pathological infertility in young women and men.

The clinical definition of infertility is the absence of conception after 12 months of regular, unprotected intercourse. Primary infertility denotes infertility of women who have never conceived, and secondary infertility denotes infertility of women who have conceived at least once and failed to conceive again.

From 1979 to 1984 the World Health Organisation (WHO) directed a worldwide study to provide a standard approach for the investigation of infertile couples (8-10). The WHO study documented that, with regards to the aetiology of infertility, 'Africa is different' compared to Latin America and Asia: i) infection was the major cause of infertility in Africa; ii) African couples had experienced longer periods of infertility before seeking treatment; iii) the prevalence of secondary 
infertility relative to primary infertility was much in Africa; and iv) the fraction of unexplained infertility was lower. Some of the strengths of the WHO study are that it developed a standard approach to diagnostic and therapeutic practice, it enrolled a large number of couples, and the study results were consistent across the centers. Unfortunately, the study centers were tertiary health care facilities that were not representative of their regions, and Africa was represented by only four centers in Ibadan (Nigeria), Lusaka (Zambia), Nairobi (Kenya) and Yaounde (Cameroon). Moreover, the study sample was restricted to infertile couples who had sought treatment and both partners had been diagnosed. Thus, it is questionable whether the type and aetiology of infertility measured in the WHO study represent the general population in all of Africa.

About $35 \%$ of infertility is due to the female, $30 \%$ to the male and $35 \%$ to the couple in western populations (11). In sub-Saharan Africa, tubal factor infertility plays a predominant role, but few studies have investigated the infertile couple and most research has focused only on the female partner (12). In addition, it is possible that the distribution of female, male, and couple factors infertility from clinic-based studies does not represent the general population. Hence, the present study aimed to investigate female, male, and couple factors infertility in both a community and a clinic-based sample.

In Tanzania, Mtimavalye et al (13) conducted an infertility study in collaboration with WHO in seven randomly selected districts in the early 1980s. This study showed that the prevalence of primary infertility was $4.3 \%$ and secondary infertility was $18.3 \%$; that primary and secondary infertility were higher in southeast compared to north-west Tanzania; and that fertile women had fewer pregnancy wastages (abortions or still births) than infertile women. Secondary data analyses of Tanzania Demographic and Health Surveys collected in the 1990s suggested that the prevalence of primary infertility was $2 \%$, secondary infertility was about $15 \%$, and that infertility varied substantially by place of residence and region (14). Further in-depth clinical studies of the type and causes of infertility in Tanzania have not been conducted.

This paper was based on infertile couples enrolled in a community-based study in Moshi Urban District (Moshi) and couples attending the out-patient clinic at Kilimanjaro Christian Medical Centre (KCMC) in Moshi for infertility treatment. Moshi had a population of 144,336 inhabitants according to the 2002 census, and it is located in the Kilimanjaro region in northern Tanzania adjacent to the Kenyan border (15). Moshi has both primary, secondary and tertiary health care facilities. It has a government regional hospital, several private hospitals, including KCMC where the clinical work for this study was done, two public health centers and numerous dispensaries with well-child and family planning services. KCMC offers referral services to the entire northern zone of Tanzania, and the Kilimanjaro region is well covered in terms of primary health care. For instance, the percentage of births delivered at a health facility was 64 in the Kilimanjaro region compared to 47 in mainland Tanzania, and the percentage of children fully vaccinated reached 94 in the Kilimanjaro region relative to 71 in mainland Tanzania (16). The objectives of this study were as follows: i) to estimate the proportion of primary and secondary infertility; ii) to assess the aetiology of infertility; iii) to determine female only factor, male only factor, female and male factors and unexplained infertility; and iv) to investigate whether the type and aetiology of infertility are different in a clinic-based and a community-based sample.

\section{MATERIALS AND METHODS}

The community-based sample of infertile couples was identified in a cross-sectional survey conducted from midNovember 2002 to end of March 2003 in Moshi. This survey interviewed a population based representative sample of 2019 women aged 20-44 years and their husbands or male partners. Following the standard clinical definition, a woman was considered to suffer from infertility if she had tried to conceive for at least 12 months, she was sexually active and not using contraception. A total of 118 couples were invited to come to KCMC for a clinical examination and possible infertility treatment and 66 (56\% of those invited) participated in the clinical investigations. The clinic-based sample included 112 couples who attended the out-patient clinic at KCMC for infertility in the period December 2002 to October 2003. All costs associated with the infertility examination and treatment, including traveling expenses to the KCMC outpatient clinic, were covered by the project, while normally infertility patients would have to pay a substantial fee for the services provided. At the first time clients visited the outpatient clinic at KCMC they were invited to participate in the infertility research project, informed about the study's objectives, the risks and benefits of participating and a consent form was signed upon entry into the clinical infertility study.

The infertility examination followed the guidelines proposed by WHO adapted to the local African situation (10). It was emphasized that an infertility examination included both the woman and the man and that the couple should attend the first clinic visit together, so that information about both partners could be collected for the medical history. However, it was common that the man refused to participate or that he completed only part of the infertility examination. In these cases only the woman entered the study, although the WHO protocol recommends that both partners in the couple participate. It was deemed unethical to exclude women from an investigation and possible treatment, even though their partners/husbands refused to participate. In addition, it could result in significant bias, if findings about the type and aetiology of infertility were based only on cases where both the female and male partners completed the infertility investigation.

The medical history included information for both the woman and the man about age, past pregnancies and children, duration of trying to get pregnant, previous contraceptive use, menstrual history and pattern, past sexually transmitted 
infections, medical and surgical history, as well as information about previous sexual partners and socioeconomic background characteristics. Following the history a pregnancy test was taken to confirm that the woman was not pregnant and subsequently both partners had a physical examination. For the woman this consisted of a general examination, a palpation of the breasts, the inspection of external and internal genitalia, and a bimanual pelvic examination. At this time the uterine size was estimated to diagnose any fibroids. The men had also a general examination, as well as a palpation of their genitalia, including the testis, epididymis and vas deferens.

Specific infertility investigations included for the women a hysterosalpingography (HSG) with fluoroscopy to determine tubal patency or pathology (unilateral or bilateral blocked tubes and uterine abnormality, such as uterine fibroid, birconuate uterus, cervical stenosis and cervical polyps), as well as a laparoscopy for those cases that had indeterminate HSG results to assess pelvic adhesions, endometriosis, unilateral or bilateral blockage and uterine abnormality. On the 21 st day of a regular menstrual cycle and for non-regular cycles on the day deemed most appropriate based on the clients menstrual pattern an endometrial biopsy was taken to assess ovulation. Serum hormone analysis is not part of the routine diagnostics used at KCMC. At first, as part of the study protocol, we attempted to collect serum for hormone analysis. Untortunately, the technicians in the laboratory were not familiar with this technique, there were problems with the laboratory testing and the hormonal tests had too many missing cases for use in the classification of ovulation. Therefore, the protocol was modified to follow the routine diagnostic services at KCMC. That is, endometrial biopsies in mid luteal phase were taken to assess endometrial lining for secretory pattern and ovulation. In addition, endometrial biopsy can detect chronic granulomatous infection of the endometrium, such as tuberculosis and schistosomiasis (infections that we do see at KCMC). Thus, the diagnosis of ovulation defect was based on one timed endometrial biopsy only.

For the men, a semen analysis was done after three days of abstinence and within two hours of semen collection. When the first analysis suggested any form of abnormality a second semen test was done following the WHO guidelines $(10,17)$. Sperm counts were categorised into four groups: i) normal sperm count included 20 million or more per $\mathrm{ml}$, ii) low normal sperm count ranged 10-19 million per $\mathrm{ml}$, iii) oligozoospermia measured less than 10 million per $\mathrm{ml}$, and iv) azoospermia (absent sperm). Morphology was classified as normal, if more than $50 \%$ normal spermatozoa. Normal spermatozoa are of ovoid head, smooth contour, acrosome visualized, normal neck, mid piece and tail. Motility and viscosity were also measured.

The subsequent diagnosis of pathological causes of infertility included female only factors (ovulation defects and tubal or uterine pathology), male only factors (oligozoospermia, azoospermia and possibly reduced motility and/or abnormal morphology), female and male factors (both partners had pathologies) and unexplained (no identified female or male factor) infertility.

The type and aetiology of infertility were analyzed separately for the community and clinic-based sample and significant differences were determined using a Pearson $\mathrm{X}^{2}$ test. Subsequently, the community and clinic-based samples were merged and the analysis was replicated on the combined sample. Risk factors for tubal pathology and ovulation defects were analyzed using univariate and multivariate logistic regression analysis and associations were presented by the relative odds ratios $(\mathrm{OR})$ and $95 \%$ confidence intervals $(\mathrm{Cl})$. The multivariate regression models included the variables associated with the dependent variable at the 0.20 level of significance.

The study protocol was approved by the Ethics Committees of KCMC and the Tanzanian National Institute for Medical Research, and the Institutional Review Board of the Harvard School of Public Health and the University of Maryland.

\section{RESULTS}

Sixty six couples entered the study from the community-based Moshi Infertility Study (MIS) and 31 $(47.0 \%)$ of these couples completed the infertility examination, i.e., the man had a semen analysis and the woman had an endometrial biopsy, an HSG and possibly a laparoscopy. The clinic-based sample entered 112 couples and $60(53.6 \%)$ couples completed the infertility examination. Thus, the sample of couples from the community and clinic-based sample, that completed the WHO guided infertility investigation, included 91 couples.

The MIS sample was restricted to Moshi. In contrast, only $56.7 \%$ of the clinic-based sample came from Moshi, while the remaining $43.3 \%$ lived in other areas of the Kilimanjaro Region or the neighbouring Arusha Region. The average (95\% confidence interval) age of the women was 30.2 years (29.3-31.1) and of the men was 35.8 years (34.7-36.9), and the age range was 20 to 43 years for the women and 20 to 52 years for the men. There was no significant difference between the community and clinic-based sample in terms of age of the woman, husband's age, religion or marital status. The majority adhered to Christianity, 26.0\% were Lutherans, $33.3 \%$ Catholics and $11.3 \%$ other Christians, while $29.4 \%$ were Muslims. Almost $70 \%$ were monogamous unions, $9.0 \%$ in polygamous unions, $19.1 \%$ cohabited, $1.7 \%$ were divorced or separated and $0.6 \%$ were widowed. The clinic-based sample had significantly more Chagga and fewer Pare than the community-based sample, that is, $47.3 \%$ versus $36.4 \%$ were Chagga and $9.8 \%$ versus $27.3 \%$ belonged to the Pare tribe, while the remaining included a vast number of different tribes.

Table 1 shows that the type of infertility was not different between the community and clinic-based sample, that $37.1 \%$ had primaly infertility and $62.9 \%$ secondary intertility. In contrast, the clinic-based sample had significantly $(\mathrm{p}=0.03)$ more childless couples, i.e., $56.3 \%$ versus $45.5 \%$, and fewer couples with at least two children, i.e. $8.9 \%$ versus $22.8 \%$. The finding that the proportion with primary infertility was not different in the community and clinic-based samples, but the clinic-based sample had significantly more childless couples suggests that the latter might 
have had more abortions. This was confirmed by the finding that $67.0 \%$ had never had an abortion in the clinic-based sample versus $74.2 \%$ in the communitybased sample. The majority of abortions were reported as miscarriages, but there was clinical evidence suggesting that some induced abortions were reported as spontaneous abortions and therefore induced and spontaneous abortions were merged into one group. The difference in the number of abortions between the community and clinic-based sample was not significant. There was also no significant difference between the two samples on how long the infertile couples had tried to conceive, and more than one third of all couples had tried to conceive for more than five years. Finally, about a half of the men reported to have children with other women suggesting that they could have been fertile at some earlier time in their reproductive life with another female.

With respect to tubal pathology we found that $25.3 \%$ had one blocked tube, $29.3 \%$ had two blocked tubes and $5.2 \%$ had uterine abnormalities (Table 2). Almost 20\% had fibroids. Ovulatory defects were found in $35.3 \%$ of the women and $9 \%$ of the women with ovulatory defects suffered from galactorrhea. Tubal factors and ovulatory defects did not differ significantly between the community and clinic-based sample. In the 133 women that completed the infertility investigation, as many as $80.5 \%$ (107 women) had some pathology and there was no significant difference between the community and clinic-based sample.

Table 1

Characteristics of infertile women in the survey and clinic sample in Moshi $(n=178)$

\begin{tabular}{|c|c|c|c|c|c|c|c|}
\hline \multirow[t]{2}{*}{ Variable } & \multicolumn{2}{|c|}{ Survey } & \multicolumn{2}{|c|}{ Clinic } & \multicolumn{2}{|c|}{ Total $^{1}$} & \multirow[b]{2}{*}{ P-value ${ }^{2}$} \\
\hline & No. & $(\%)$ & No. & $(\%)$ & No. & $(\%)$ & \\
\hline Type of infertility & & & & & & & 0.64 \\
\hline Primary & 23 & 34.9 & 43 & 38.4 & 66 & 37.1 & \\
\hline Secondary & 43 & 65.2 & 69 & 61.6 & 112 & 62.9 & \\
\hline Parity & & & & & & & 0.03 \\
\hline 0 & 30 & 45.5 & 63 & 56.3 & 93 & 52.3 & \\
\hline 1 & 21 & 31.8 & 39 & 34.8 & 60 & 33.7 & \\
\hline $2-3$ & 12 & 18.2 & 10 & 8.9 & 22 & 12.4 & \\
\hline $4-5$ & 3 & 4.6 & 0 & 0 & 3 & 1.7 & \\
\hline \multicolumn{8}{|c|}{ Duration of infertility (years) } \\
\hline$<2$ & 7 & 10.9 & 23 & 20.7 & 30 & 17.1 & \\
\hline 2 & 11 & 17.2 & 19 & 17.1 & 30 & 17.1 & \\
\hline 3 & 13 & 20.3 & 20 & 18.1 & 33 & 18.9 & \\
\hline 4 & 4 & 6.3 & 12 & 10.8 & 16 & 9.1 & \\
\hline $5-9$ & 20 & 31.3 & 26 & 23.4 & 46 & 26.3 & \\
\hline $10-20$ & 9 & 14.1 & 11 & 9.9 & 20 & 11.4 & \\
\hline Number of abortions & & & & & & & 0.59 \\
\hline 0 & 49 & 74.2 & 75 & 67.0 & 124 & 69.7 & \\
\hline 1 & 10 & 15.2 & 21 & 18.8 & 31 & 17.4 & \\
\hline $2-5$ & 7 & 10.6 & 16 & 14.3 & 23 & 12.9 & \\
\hline $\begin{array}{l}\text { Husband has children } \\
\text { with other women }\end{array}$ & & & & & & & 0.22 \\
\hline No & 28 & 43.1 & 58 & 52.7 & 86 & 49.1 & \\
\hline Yes & 37 & 56.9 & 52 & 47.3 & 89 & 50.9 & \\
\hline Total & 66 & 37.1 & 112 & 62.9 & 178 & 100 & \\
\hline
\end{tabular}

${ }^{1}$ For some variables the sample size does not add up to the total because of missing cases

2 Based on a Pearson $\mathrm{X}^{2}$ test 
Table 2

Clinical diagnosis of women in the survey and clinic sample in Moshi

\begin{tabular}{|c|c|c|c|c|c|c|c|}
\hline \multirow[t]{2}{*}{ Variable } & \multicolumn{2}{|c|}{ Survey } & \multicolumn{2}{|c|}{ Clinic } & \multicolumn{2}{|c|}{ Total } & \multirow[b]{2}{*}{ P-value ${ }^{1}$} \\
\hline & No. & $(\%)$ & No. & $(\%)$ & No. & $(\%)$ & \\
\hline Tubal disease $(\mathrm{n}=174)$ & & & & & & & 0.92 \\
\hline Patent tubes & 24 & 38.1 & 46 & 41.4 & 70 & 40.2 & \\
\hline Unilateral blockage & 16 & 25.4 & 28 & 25.2 & 44 & 25.3 & \\
\hline Bilateral blockage & 20 & 31.8 & 31 & 27.9 & 51 & 29.3 & \\
\hline Uterine abnormality & 3 & 4.8 & 6 & 5.4 & 9 & 5.2 & \\
\hline Uterine size $(n=176)$ & & & & & & & 0.26 \\
\hline Fibroids & 16 & 24.2 & 19 & 17.3 & 35 & 19.9 & \\
\hline Ovulation $(n=133)$ & & & & & & & 0.53 \\
\hline Defect $^{2}$ & 16 & 32.0 & 31 & 37.4 & 47 & 35.3 & \\
\hline Defect and galactorrhoea & 1 & 2.0 & 11 & 13.3 & 12 & 9.0 & 0.03 \\
\hline
\end{tabular}

${ }^{1}$ Based on a Pearson $\mathrm{X}^{2}$ test

${ }^{2}$ Endometrial biopsy suggested irregular proliferation, no secretion, atrophy or other

There was no significant difference between the community- and clinic-based samples in terms of abnormal sperm (Table 3). A total of $15.6 \%$ of the men suffered from oligozoospermia and $7.3 \%$ had azoospermia, i.e., a total of $22.9 \%$ of the men had abnormal sperm. As many as $7.3 \%$ had a low normal sperm caunt of $10-19$ million per ml, although there is ample evidence suggesting that the latter men may be able to make a woman pregnant. More than $10 \%$ had reduced or no motility and $7.3 \%$ had abnormal or no morphology. Each of the cases with poor motility, morphology and/or viscosity suffered from either oligozoosperma of azoospermia.

Table 3

Clinical diagnosis of male partners of women in the survey and clinic sample in Moshi $(n=109)$

\begin{tabular}{|c|c|c|c|c|c|c|c|}
\hline \multirow[t]{2}{*}{ Variable } & \multicolumn{2}{|c|}{ Survey } & \multicolumn{2}{|c|}{ Clinic } & \multicolumn{2}{|c|}{ Total } & \multirow[b]{2}{*}{ P-value ${ }^{1}$} \\
\hline & No. & $(\%)$ & No. & $(\%)$ & No. & $(\%)$ & \\
\hline Sperm count & & & & & & & 0.50 \\
\hline Normal & 28 & 77.8 & 48 & 65.8 & 76 & 69.7 & \\
\hline Low normal & 2 & 5.6 & 6 & 8.2 & 8 & 7.3 & \\
\hline Oligozoospermia & 5 & 13.9 & 12 & 16.4 & 17 & 15.6 & \\
\hline Azoospermia & 1 & 2.8 & 7 & 9.6 & 8 & 7.3 & \\
\hline Motility & & & & & & & 0.34 \\
\hline Normal & 33 & 91.7 & 64 & 87.7 & 97 & 89 & \\
\hline Reduced & 0 & 0 & 4 & 5.5 & 4 & 3.7 & \\
\hline Non motile & 3 & 8.3 & 5 & 6.8 & 8 & 7.3 & \\
\hline Morphology & & & & & & & 0.40 \\
\hline Normal & 35 & 97.2 & 66 & 90.4 & 101 & 92.7 & \\
\hline Abnormal & 0 & 0 & 2 & 2.7 & 2 & 1.8 & \\
\hline No sperm & 1 & 2.8 & 5 & 6.8 & 6 & 5.5 & \\
\hline Viscosity & & & & & & & 0.37 \\
\hline Normal & 33 & 91.7 & 69 & 94.5 & 102 & 93.6 & \\
\hline Increased & 2 & 5.6 & 4 & 5.5 & 6 & 5.5 & \\
\hline Reduced & 1 & 2.8 & 0 & 0 & 1 & 0.9 & \\
\hline \multicolumn{8}{|l|}{ Male factor ${ }^{2}$} \\
\hline Normal & 30 & 83.3 & 54 & 74 & 84 & 77.1 & \\
\hline Abnormal & 6 & 16.7 & 19 & 26 & 25 & 22.9 & \\
\hline
\end{tabular}

${ }^{1}$ Based on a Pearson $\mathrm{X}^{2}$ test

2 Abnormal includes at least one pathology 
A total of 91 couples completed the entire infertility investigation $(51.1 \%$ of the 178 couples that participated in the clinical investigations). That is, both partners gave a medical history, both had a general examination, the man had a semen analysis, the woman had a hysterosalpingogram, possibly also a laparoscopy, and an endometrial biopsy. Based on this sample the distribution of female and male factor infertility was a follows: female and male factors infertility included $13.2 \%$ (12 cases), only female factor $65.9 \%$ (60 cases), only male factor $8.8 \%$ (8 cases) and unexplained infertility $12.1 \%$ (11 cases) (Figure 1$)$.

\section{Figure 1}

The distribution of female, male, female and male and unexplained infertility

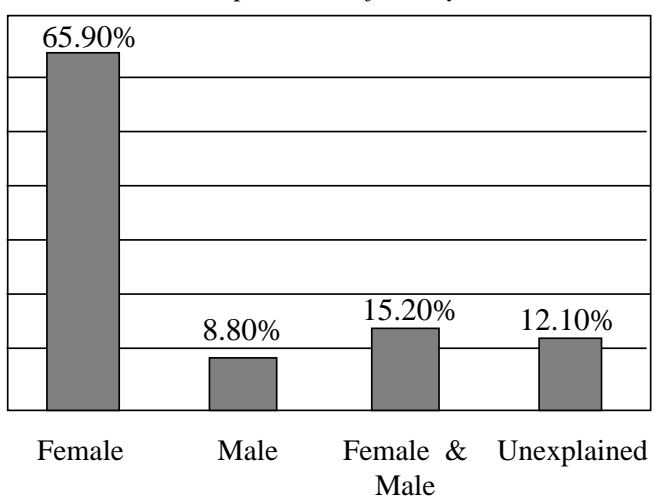

Table 4

The associations between past STDs, abortions, complications at last delivery and other reproductive characteristics and tubal pathology in total sample in Moshi $(n=174)$

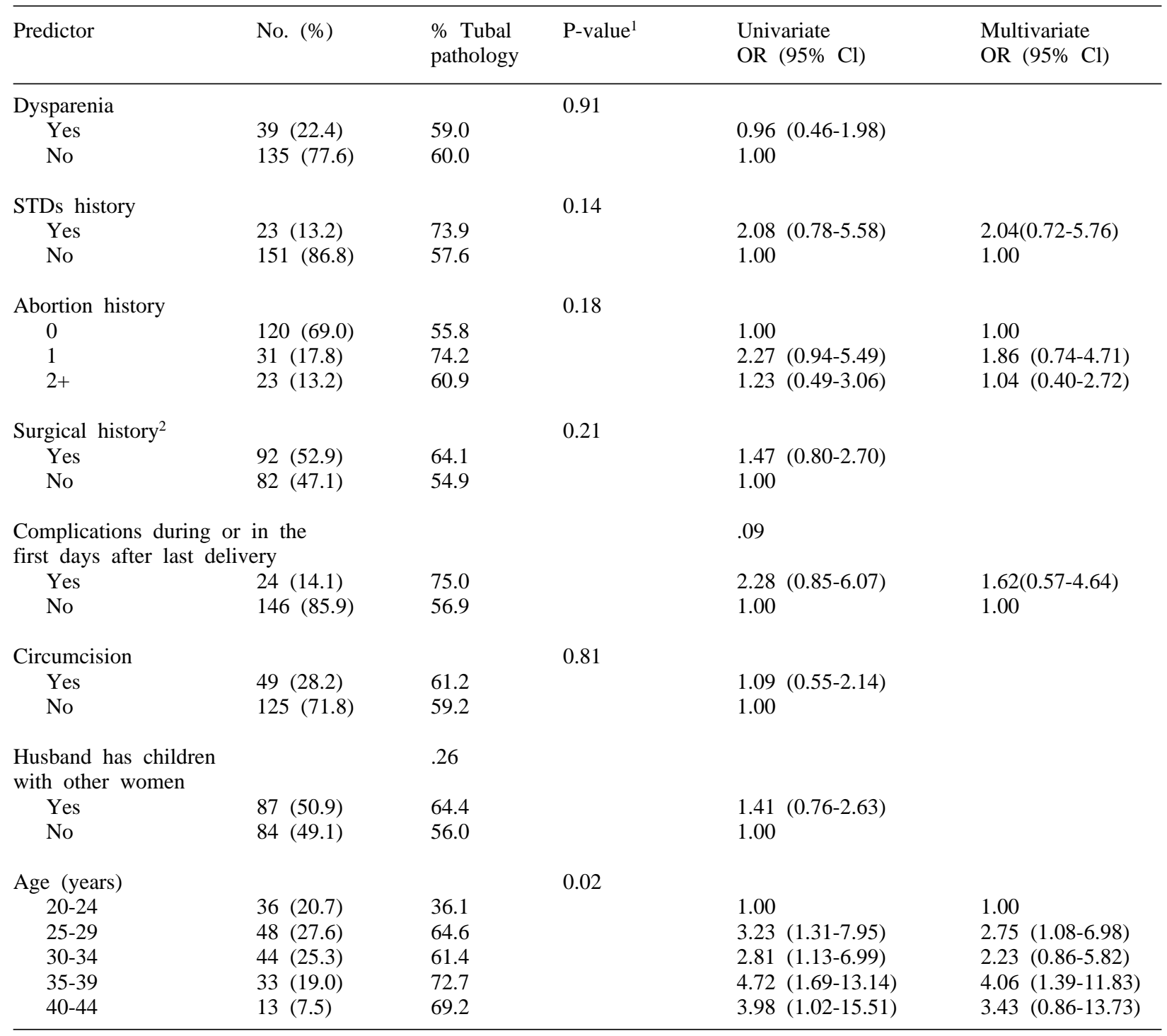

\footnotetext{
${ }^{1}$ Based on a Pearson $\mathrm{X}^{2}$ test

2 Surgeries included myomectomy, D \& C, appendectomy, ectopic pregnancy and Caesarian section
} 
Table 5

The associations between menstrual characteristics and ovulation defects in total sample in Moshi (n = 133)

\begin{tabular}{|c|c|c|c|c|}
\hline Predictor & No. $(\%)$ & $\begin{array}{l}\% \text { Ovulation } \\
\text { defect }\end{array}$ & P-value ${ }^{1}$ & $\begin{array}{c}\text { Univariate } \\
\text { OR }(95 \% \mathrm{Cl})\end{array}$ \\
\hline Menstrual cycle & & & 0.06 & \\
\hline Regular & $110(82.7)$ & 31.8 & & 1.00 \\
\hline Irregular & $23(17.3)$ & 46.2 & & $2.34(0.94-5.81)$ \\
\hline Dysmenorhoea & & & 0.31 & \\
\hline Yes & $84(63.2)$ & 32.1 & & $0.69(0.33-1.43)$ \\
\hline No & $49(36.8)$ & 40.8 & & 1.00 \\
\hline Fibroids & & & 0.41 & \\
\hline Yes & $25(19.1)$ & 28.0 & & $0.67(0.26-1.74)$ \\
\hline No & $106(80.9)$ & 36.8 & & 1.00 \\
\hline Age (years) & & & 0.67 & \\
\hline $20-24$ & $24(18.1)$ & 33.3 & & 1.00 \\
\hline $25-29$ & 38 (28.6) & 36.8 & & $1.17(0.40-3.42)$ \\
\hline $30-34$ & 38 (28.6) & 42.2 & & $1.45(0.50-4.22)$ \\
\hline $35-39$ & $22(16.5)$ & 22.7 & & $0.59(0.16-2.18)$ \\
\hline $40-44$ & $11(8.3)$ & 36.4 & & $1.14(0.26-5.09)$ \\
\hline
\end{tabular}

${ }^{1}$ Based on a $\mathrm{X}^{2}$ test

In the sub-sample of 91 couples that completed the entire infertility investigation as many as $45.1 \%$ had primary infertility and $61.5 \%$ were childless compared to $37.1 \%$ and $52.3 \%$ respectively in the 178 couples that entered the study. Thus, couples with primary infertility and childless couples were more likely to complete the infertility examination, as one would expect, because they had never experienced parenthood and their desire for a child were greater. However, if the analysis of the type of infertility and the characteristics of infertile couples were restricted to the couples who had completed the infertility examination, then one would falsely conclude that primary infertility and childlessness included a relatively higher proportion of total infertility.

Table 4 lists the distribution of selected risk factors for tubal pathology, such as sexually transmitted diseases (STDs) history, abortion history, complications during or in the first days after last delivery, circumcision and surgical history. The associations between tubal pathology and each risk factor were attenuated in the multivariate model. Tubal pathology was higher for women who reported an STDs history, one abortion, and complications during or in the first days after last delivery, although none of these associations were significant at the 0.05 level. Finally, the higher the age of the woman the higher the risk of tubal pathology.

Women with irregular cycles were 2.34 times more likely to be diagnosed with ovulation defects $(p=0.06)$ according to endometrial biopsies (Table 5). In contrast, there was no association between dysmenorrhoea, fibroids and age.

\section{DISCUSSION}

This study analysed the type and aetiology of infertility in a community and clinic-based sample in Moshi in northern Tanzania. Three main findings were derived from this work. For one, the type and aetiology of infertility were the same in the community and clinic-based sample suggesting that the couples seeking infertility health care were representative of the general infertile population, at least in this urban population in northern Tanzania. Primary infertility affected about one third and secondary infertility about two thirds of couples with infertility. Female only factors accounted for as much as $65.9 \%$, male only factors $8.8 \%$, female and male factors $13.2 \%$ and unexplained infertility $12.0 \%$. Thus, women carried a much higher fraction of the burden of infertility in this urban population in northern Tanzania in comparison to the pattern of about $35 \%$ female factor, $30 \%$ male factor and $35 \%$ couple factors often found in western populations (12).

Second, it proved very difficult to get the male partners in the infertile couples to participate and to complete the infertility investigation. Specifically, the men were reluctant to provide a sperm sample for testing of sperm quality, and the men were particularly reluctant to provide a second sample in the cases that had suggested sperm abnormalities in the first test. As a result only about a half of the couples who entered the infertility investigation completed the infertility work up. This lack of male participation is a serious barrier to providing the best standard of care to infertile couples. Thus, efforts to raise awareness in the population about the causes of infertility are needed and educational materials about infertility could be linked to ongoing efforts aimed at reducing high risk sexual behaviour, such as the Abstinence, Be faithful and use Condoms (ABC) campaigns. The finding that the male partner is very underrepresented among health care seekers for infertility might contribute to an underestimate of male factor infertility and to the tendency of blaming the woman for the condition of infertility. In addition, 
inferences about the type of infertility might be biased because, as was the case in this analysis, the couples where both partners sought care were more likely to suffer from primary infertility and to be childless. SubSaharan African populations in general are pronatalist and the quest for parenthood is very strong making childless men more amenable to seeking infertility treatment. Thus, for example the WHO study might have under-estimated the proportion of secondary infertility by restricting the analysis to only couples who had completed the entire infertility investigation and received a diagnosis, although the WHO study was the first to emphasize that secondary infertility was relatively more prevalent in sub-Saharan Africa compared to Latin America and Asia (8). The social consequences of infertility are often marital dissolution or extra-marital sexual relations, and increased exposure to STIs/HIV, as well as psychological impairments (1). Each of these factors surfaced in the clinical histories suggesting that health care services for infertility should include couple counselling.

The third finding was that even if, as expected in Africa, a majority suffered from tubal factor infertility, ovulation defects were also prevalent. There were unprecedented high percentages of infertile women presenting with galactorrhoea $(9.0 \%)$ and uterine fibroids $(19.1 \%)$. Galactorrhoea is one specific manifestation of hormonal dysfunction, suggesting anovulation and hyperprolactinaemia. We do not know the reason for the high level of this condition in this sample. Also, hormonal function was not assessed in almost a quarter of the sample making inferences about hormonal dysfunction tentative. Uterine fibroids are common in African women, but almost $20 \%$ in a population of young women in their most fertile age range was unexpectedly high (6). Further research on the causes of fibroids are in order, as well as the association between the clinical management of fibroids and subsequent infertility.

In general, some women with ovulation defects have children following treatment with, for example, clomiphene, while tubal surgeries for tubal blockage yield very low pregnancy rates and other treatment options are not available in Moshi in northern Tanzania or in most other sub-Saharan African settings. Recently, modern reproductive technologies have been applied successfully in Nigeria and South Africa, but for most African populations such services will not be manageable in the foreseeable future $(6,18,19)$. Public health efforts aimed at preventing onset of infertility in young women and men are still the best option in terms of helping African couples having the number of children they desire and at the time they so desire.

\section{ACKNOWLEDGEMENTS}

This project was funded by the National Institute of Child Health and Human Development (NICHD R01 HD41202). Johanne Sundby provided valuable comments on an early version of this paper. We would like to thank the nurses and other staff at KCMC for their contribution to the clinical management of infertile couples and data collection. Finally, we want to express our sincere appreciation to all the infertile women and men who shared their infertility experiences and participated in this study.

\section{REFERENCES}

1. Boerma, J.T. and Mgalla, Z. (Eds). Women and Infertility in sub-Saharan Africa: A Multi-disciplinary perspective. Royal Tropical Institute, KIT Publishers, Amsterdam, 2001.

2. Inhorn, M.C. and van Ballen, F. (Eds). Infertility around the globe: New thinking on childressness, gender and reproductive technologies. University of California Press, Berkeley, 2001.

3. Bentely, G.R. and Mascie-Taylor, C.G.N. (Eds). Infertility in modern world: Present and future prospects. Cambridge University Press, Cambridge. UK, 2000.

4. Inhorn, M.C. Babies in global test tubes: Gender, science, and reproductive technology in Islamic Egypt. Routledge, New york, 2004.

5. Menken, J. and Larsen, U. Infertility, demographic aspects of Entry 3.3.86 in International Encyclopedia of the Social and Behavioral Sciences. Elsevier Science, Cambridge, 2002.

6. Okonofua, F., Menakaya, U., Iribhogbe, P., et al. Aetiology and outcome of treatment in 190 infertile Nigerian couples. Unpublished manuscript.

7. Ajayi, R.A., Parsons, J.H. and Bolton, V.N. Live births after intracytoplasmic sperm injection in the management of oligospermia and azoospermia in Nigeria. Afr. J. Reprod. Health. 2003; 7: 121-124.

8. Cates, W., Farley, T.M.M. and Rowe, P.J. Worldwide patterns of infertility: Is Africa different? Lancet. 1985; 14: 596-598.

9. WHO. Infections, pregnancies, and infertility: Perspectives on prevention. Fertility and Sterility. 1987; 47: 964-968.

10. Rowe, P.J., Comhaire, F.H., Hargreave, T.B. and Mellows, H.J. WHO Manual for the Standard Investigation and Diagnosis of the Infertile Couple. Cambridge University Press, Carnbridge, 1993.

11. Sherris, J. and Fox, G. Infertility and sexually transmitted disease: A public health challenge. Population Reports Series L. 1983; 4: L113-L151.

12. Mayaud, P. The role of reproductive tract infections. In: Women and Infertility in Sub-Saharan Africa: A Multidisciplinary Perspective, Boerma, J.T. and Mgalla, Z. (Eds). Royal Tropical Institute, KIT Publishers, Amsterdam, 2001:71-109.

13. Mtimavalye, L.A.R., Masawe, F.N., Remme. J. et al. Infertility among women in five rural and two urban districts of Tanzania. J. Obstet. Gyn. East and Central Africa. 1984; 3: 125-129.

14. Larsen, U. and Raggers, H. Levels and trends in infertility in sub-Saharan Africa. In: Women and Infertility in SubSaharan Africa: A Multi-disciplinary Perspective, Boerma, J.T. and Mgalla, Z. (Eds). Royal Tropical Institute, KIT Publishers, Amsterdam, 2001; 25-70.

15. Website: http://www.tanzania.go.tz/census

16. 1996 DHS the Bureau of Statistics [Tanzania] and Macro International Inc. 1997. Tanzania Demographic and Health Survey 1996. Calverton, Maryland: Bureau of Statistics and Macro International. Pp. 110, 116.

17. WHO laboratory manual for the examination of human Semen and sperm-cervical mucus interaction. 4th edition, Cambridge: Cambridge University Press, 1999.

18. Okonofua, F. New reproductive technologies and infertility treatment in Africa. Afr. J. Reprod. Health. 2003; 7: 7-8.

19. Curent practices and controversies in assisted reproduction. Report of a meeting on "Medical, Ethical and Social Aspects of Assisted Reproduction". WHO Headquarters, Geneva, Switzerland, 17-21 September 2001. 